\title{
Allergen-specific immunotherapy: an update on immunological mechanisms of action
}

\author{
G. Ciprandi1, G.L. Marseglia², M.A. Tosca³
}

ABSTRACT: Allergen-specific immunotherapy: an update on immunological mechanisms of action. G. Ciprandi, G.L. Marseglia, M.A. Tosca.

Specific immunotherapy (SIT) is the only treatment able to modify the natural history of the allergic subjects. Several aspects of the immunopathological response modified by SIT have been investigated; the first parameter historically studied was the production of allergen-specific antibodies. An increase of allergen-specific IgG4 and a decrease of IgE appear after SIT. A shift from Th2-polar- ized immune response toward Th1-oriented pattern has been reported after SIT. More recently, a crucial role for a subpopulation of $\mathrm{T}$ cells has been evidenced: $\mathrm{T}$ regulatory cells (Treg). Allergic patients have a defect of Tregs. SIT is able of inducing a specific Treg response.

Sublingual immunotherapy is an alternative route of administration for SIT. Recent evidence shows that SLIT is also able of inducing a Treg response as detected by IL10 production.

Monaldi Arch Chest Dis 2006; 65: 1, 34-37.

Keywords: Allergen-specific immunotherapy, atopy, $\operatorname{IgE}, \operatorname{IgG}_{4}$.

${ }^{1}$ Azienda Ospedaliera Universitaria "San Martino", Genova

2 IRCCS San Matteo Pavia e Università di Pavia,

3 Istituto Giannina Gaslini, Genova, Italy.

Correspondence: Giorgio Ciprandi, M.D.; Allergologia - U.O. ORL; Dipartimento Patologie Testa-Collo; Padiglione Specialità (piano terzo); A.O.U. San Martino; Largo R. Benzi 10, 16132 Genoa, Italy; e-mail gio.cip@libero.it

\section{Introduction}

Specific immunotherapy is the only treatment able to modify the natural history of allergic subjects. Immunotherapy creates a "clinical tolerance" towards an allergen that causes a specific clinical picture and may inhibit the progressive evolution both towards polysensibilization and towards asthma-rhinitis comorbidity $[1,2,3,4,5]$.

Most of the studies published so far, focus on subcutaeous specific immunotherapy (SCIT); recently, though, a few clinical studies have investigated alternative ways, mainly the sublingual one (SLIT).

\section{SCIT Mechanisms}

Several aspects of the immunopathological response modified by SCIT have been investigated; the first parameter historically studied was the production of allergen-specific antibodies.

\section{Effects on the Synthesis of Allergen-specific Anti-} bodies

The concentration of specific serum $\operatorname{IgE}$ increases during the initial phases of SCIT, and later decreases over a period of time that varies from weeks to several months, until it reaches lower values than at the beginning. As a result, the immediate response to skin tests may be reduced. On the other hand, the late phase to skin tests is virtually abolished, as well as the late phase of nasal, ocular, and bronchial response to the allergen-specific challenge. Reduced IgE production could be due to different mechanisms, none of which have been definitively confirmed. The partial or total inhibition of cytokines activating the mediated $\mathrm{IgE}$ response is probably the most reliable hypothesis [6].

During specific immunotherapy, the IgG class increases, mainly concerning the $\mathrm{IgG}_{4}$ subclass. These antibodies, having a high affinity with the specific allergen, which compete against the IgE (hence the term "blockers", as they prevent the allergen from reaching the target cell in the reaction organ; they indeed block the activation of the mast-cells and inhibit the release of mediators). The $\mathrm{IgG}_{4}$ are produced by B cells, in presence of both IFN $\gamma$ and IL-10.

Besides inhibiting the release of chemical mediators from mast cells and basophil cells, these antibodies can also prevent the infiltration of inflammatory cells in the target organs which results in a reduction in number of mast cells and eosinophils, an inhibition of their activation, and a reduced release of histamine, $\mathrm{PGD}_{2}$, and other mediators, leading to an inhibition of the late allergic response.

\section{Effects on Th1 and Th2 Lymphocytes}

As for the Th1 and Th2 balance, it has been demonstrated that immunotherapy modifies the patient's allergic phenotype, generally characterised 
by a Th2 polarization: in fact, immunotherapy has been demonstrated to stimulate a Th1-type response ("immunodeviation") related to an increased IFN $\gamma$ and IL-2 production. This response is promoted and strengthened, thanks to activated macrophages, by an increased IL-12 synthesis, which powerfully stimulates the IFN $\gamma$ synthesis [7].

The effectiveness of SCIT could be due to a Th2 reduced activity, either through a mechanism of anergy or, at least, of tolerance ("immunosuppression") or through a Th1 stimulation and regulation (also known as "immune deviation") $[8,9]$. In fact, high doses of allergen, as provided by immunotherapy, could induce an immunological tolerance. In patients receiving SCIT a significant reduction of the in vitro specific allergen inducedproliferation of $\mathrm{CD} 4+$ T-lymphocytes has been demonstrated, as well as a reduced production of IL-4 and IL-5. Moreover, specific immunotherapy is able to reduce the expression of ICAM-1 adhesion molecules on the nasal and conjunctival epithelium, the infiltrate of inflamatory cells, and the plasmatic level of IL-13 [10, 11].

During immunotherapy for hymenoptera venom and for inhalant allergens, a significant induction of a sub-popoulation of T-cells, the T-regulatory cells (Treg), whose activation is fundamental for the homeostasis regulation has been recently described $[12,13,14,15]$.

\section{Role Played by Regulatory T-cells}

The context in which Treg cells, divided into Tr1, Th3, CD $4+$ CD $25+$ (the $\alpha$ chain of the IL-2 receptor, IL-2R $\alpha$ ), are generated has not yet been well defined. Furthermore, the mechanism through which Treg cells suppress the T-cell mediated immune response still needs to be clarified; nevertheless, the immunosuppressant and/or immunoregulatory contribution of their cytokines IL-10 and TGF $\beta$ is nowadays of great interest [16], since their increased production is essential for the immunotherapy effectiveness.

Interleukin $10(18.7 \mathrm{Kda})$ plays a role in a complex series of reactions during the allergic response; produced by $\operatorname{Tr} 1$, Th1, Th2, mononuclear macrophages and NK cells, it determines a prolonged inhibition of the allergen-specific T CD4+ response, reduces the number of resident mast cells and the production, the recruitment, and the increased survival of the eosinophils [17]. Moreover, it reduces the activation of allergen-specific Th2 cells, the production of pro-inflammatory cytokines by macrophages, and cytokines released by $\mathrm{IgE}$-activated mast cells; it also reduces the $\mathrm{IgE}$ production while increasing the synthesis of IgG4 by B-lymphocytes [4]; IL-10 also suppresses the synthesis of cytokines produced by T-cells (IFN $\gamma$, IL2, IL4, IL5, IL6, IL8, IL12, TNF $\alpha$ ), the synthesis of both CD23 and ICAM-1, and it even reduces the expression of co-stimulation signals between CD28 (present on T helper lymphocytes) and B7 (present on antigen presenting cells, APC); finally, it inhibits the APC-mediated production of accessory signals for the activation of $\mathrm{T}$ helper cells.
At the target organ, IL-10 inhibits the proliferation of the vascular smooth muscle, with a possible benefial effect of reducing the remodeling of the respiratory tract in patients affected by asthma.

TGF $\beta$ is another cytokine implicated in the modulation of the allergic reaction. It is produced by Treg cells [11, 17, 18, 19]. A significant effect can be detected in the increased production of secretory $\operatorname{Ig} \mathrm{A}$, through the activation of $\mathrm{B}$ cells, in the inhibition of both the GATA 3 expression and the related orientation towards the Th2 and Th1 population, and in the crucial role played in the induction of the tolerance for the oral antigen presentation.

It is possible that all the complex mechanisms just described could take part in immunotherapy, by the activation of the Treg subpopulation. In fact, the anergy of the allergen-specific $\mathrm{T}$ cells deriving from this treatment, if occurring, seems to be al least partly dependent on the action of the IL10 [19]. Also, as for the IL-10, two recent papers $[20,21]$ underline its fundamental role in inducing an anergy as a consequence of SCIT: in particular, this cytokine seems to be able to predict the success of the specific treatment, when an increase in IL10-mRNA is detected after 3 month administration. On the other hand, the TGF $\beta$ will increase after one year, but only in the responding subjects. Another cytokine, the IL-18 increases during SCIT, especially in the first phases; in presence of the co-stimulator cytokine IL-12. IL-18 favours both the production of IFN $\gamma$ and the Th1 switch, and it is an antagonist of the Th2 response, through the production of $\operatorname{IgE}$ and the hyperreactivity of the respiratory tract.

\section{Role of Dendritic Cells}

Mucosal dendritic cells of the respiratory tract and of the digestive tube control the first phase ("mucosal") of the immunological response, by capturing and elaborating the allergens, followed by the second systemic phase that sees the activation of a process leading to the Th1 polarization or to a response of allergen-specific immunotolerance. The role of the Dendritic Cells (DC) becomes thus fundamental [8].

Immunotherapy with high doses of allergen may give different levels of response, both for dendritic and regulatory cells. This is true mainly for sublingual exhibition at high antigen doses, rapidly captured and sent to the DC of the draining lymph nodes, and then exposed to the T-cells, thanks to IL-12, in order to activate a process that will bring to Th1 polarization. Followed by lymphocytes re-circulation and allergen absorption, this mechanism becomes similar to SCIT (specific IgE increase, specific $\mathrm{T}$ proliferative response, etc.) through a mechanism of immunodeviation or, more properly, of immunosuppression.

On the whole, specific immunotherapy has a significant effect on modulating allergen-specific T-cells: this fact explains why both the clinical and the late response are attenuated, even if specific serum antibodies are not directly involved at the same time. 
Most of the recent approaches of specific immunotherapy aim at obtaining a switch to a physiologic Th1 oriented asset [8], mainly through the induction of regulatory T cells and IL-10 and TGF $\beta$ production $[22,23]$.

\section{SLIT Mechanisms}

As for SLIT, in order to be efficient, as recently underlined in the ARIA document, the doses of allergen have to be really high, such as they have to be superior to those used for the subcutaneous injection (from 50 up to 100 times the cumulative doses of SCIT) [1]. Another consideration that has to be made concerns the allergen kinetics. After having been underneath the tongue for a short period of time and then swallowed, it may be rapidly and almost completely detected in the stomach and the small intestine, while a small portion of it, up to $2 \%$, remains in the sublingual region for up to 20-30 hours [24]. On the other hand, the plasmatic radioactivity reaches a peak after 1 hour, and persists after 2 hours, demonstrating that the absorption takes place mainly in the gastroenteric tract. Actually, the high molecular weight of allergen extracts wouldn't allow a sublingual absorption.

As previously mentioned, very few studies have investigated the immunologic mechanism of this type of administration and, so far, only three parameters have been evaluated: allergen-specific antibodies synthesis, in vitro cytokine production and proliferative response.

As for the first parameter, data published so far seems to be contrasting, since some authors have found an increased synthesis of specific IgG4, while others haven't demonstrated this particular phenomenon [24]. Of course, this data discrepancy could depend on several methodological aspects that may indeed differentiate the studies (i.e. type of allergen, concentrations, duration of treatment, etc.).

As for the cytokines synthesis, so far only one paper has been able to detect a reduced level of IL13 , even though in a in vitro model [11].

Furthermore, one study has reported that SLIT is able to induce a reduced proliferative allergenspecific response, which indirectly demonstrates the involvement of a regulatory response [25].

At last, very recent data has shown that in patients treated for 3 years with successful SLIT a reduced proliferative response is detectable, in addition an increased production of IL-10 is present only in SLIT-treated allergic patients [26, 27]. This fact directly demonstrates the involvement of the regulatory response as a consequence of sublingual assumption of the allergenic extract. In addition, it has been reported that the reduction of eosinophilic infiltration and bronchial hyperreactivity are related with IL-10 production in SLITtreated allergic patients [28].

\section{Conclusions}

Specific immunotherapy is at present the only therapeutic strategy oriented towards the allergen specific response. Moreover, it represents the only treatment able to modify the natural history of the allergic reaction, preventing the typical evolution to polysensitization, the symptom aggravation, and the asthma-rhinitis association. Its efficacy has been widely demonstrated. Moreover, specific immunotherapy is the only specific treatment for respiratory allergies, since no other treatment can carry on a similar immunomodulant activity against the causal allergen. Furthermore, in order to assure a therapeutic benefit, the diagnosis must be correct and precise. Especially in polysensitised patients, the main allergen responsible for the major symptoms has to be detected. It is still important, though, to consider that appropriate therapeutic management of the allergic patient should be based on a multidisciplinar approach where the allergist is the physician who should actually unify the various professional aspects.

\section{References}

1. Lockey RF. "ARIA": Global guidelines and new forms of allergen immunotherapy. J Allergy Clin Immunol 2001; 108: 497-9.

2. Lewis DB. Allergy immunotherapy and inhibition of Th2 immune responses: a sufficient strategy? Curr Opin Immunol 2002; 14: 644-51.

3. Waldmann TA. Immunotherapy: past, present and future. Nat Med 2003; 9: 269-77.

4. Frew AJ. Immunotherapy of allergic disease. J Allergy Clin Immunol 2003; 111: S712-9.

5. Fasce L, Tosca MA, Olcese R, Milanese M, Erba D, Ciprandi G. The natural history of allergy: the development of new sensitisations in asthmatic children. Immunology Letters 2004; 93: 45-50

6. Busse WW, Rosenwasser LJ. Mechanisms of asthma. $J$ Allergy Clin Immunol 2003; 111: S799-804.

7. Durham SR, Till SJ. Immunological changes associated with allergen immunotherapy. J Allergy Clin Immunol 1998; 102: 157-64.

8. Romagnani S. New therapeutic strategies in allergic diseases. Drugs of Today 2003; 39: 849-65.

9. Frieri M. New frontiers in immunotherapy. Allergy Asthma Proc 1999; 20: 281-8.

10. Canonica GW, Passalacqua G. Noninjection routes for immunotherapy. J Allergy Clin Immunol 2003; 111: 437-48.

11. Ippoliti F, De Sanctis W, Volterrani A, et al. Immunomodulation during sublingual therapy in allergic children. Ped Allergy Immunol 2003; 14: 216-21.

12. Bellinghausen I, Metz G, Enk AH, Christmann S, Knop J, Saloga J. Insect venom immunotherapy induces IL10 production and a Th2 to Th1 shift, and changes surface marker expression in venom-allergic subjects. Eur $J$ Immunol 1997; 27: 586-96.

13. Nasser SM, Ying S, Meng Q, Kay AB, Ewan PW. IL10 levels increase in cutaneous biopsies of patients undergoing wasp venom immunotherapy. Eur J Immunol 2001; 31: 3704-13.

14. Akdis CA, Blesken T, Akdis M, Wutrich B, Blaser K. Role of IL10 in specific immunotherapy. J Clin Invest 1998; 102: 98-106.

15. Akdis CA, Blaser K. IL10 induced anergy in peripheral $\mathrm{T}$ cells and reaction by microenvironmental cytokines: two key steps in specific immunotherapy. FASEB $J$ 1999; 13: 603-9.

16. McHugh RS, Shevah EM. The role of suppressor T cells in regulation of immune responses. J Allergy Clin Immunol 2002; 111: 693-702. 
17. Bellinghausen I, Knop J, Saloga J. The role of IL10 in the regulation of allergic immune responses. Int Arch Allergy Immunol 2001; 126: 97-101.

18. Schramm C, Herz U, Podlech J, et al. TGFbeta regulates airway responses via T cells. J Immunol 2003; 170: 1313-9.

19. Koulis A, Robinson DG. The anti-inflammatory effects of IL10 in allergic disease. Clin Exp Allergy 2000; 30: $747-50$

20. Blaser K, Akdis CA. IL10, T regulatory cells and specific allergy treatment. Clin Exp Allergy 2004; 34: 328-31.

21. Savolainen J, Laaksonen K, Rantio-Lehtimski G, Terho EO. Increased expression of allergen-induced in vitro IL10 and IL18 mRNA in peripheral blood mononuclear cells of allergic rhinitis patients after specific immunotherapy. Clin Exp Allergy 2004; 34: 413-9.

22. Homer AA, Van Uden JH, Zubeldia JM, Broide D, Raz E. DNA-based immunotheraputics for the treatment of allergic disease. Immunol Rev 2001; 179: 102-18.

23. Jutel M, Akdis M, Budak F, et al. IL10 and TGFbeta cooperate in the regulatory $\mathrm{T}$ cell response to mucosal allergens in normal immunity and specific immunotherapy. Eur J Immunol 2003; 33: 1205-14.

24. Passalacqua G. Immunoterapia specifica. In: Zanussi C (Ed). Trattato Italiano di Allergologia. Pavia: Selecta Medica 2002: 1055-73.

25. Fanta C, Bohle B, Hirt W, et al. Systemic immunological changes induced by administartion of grass pollen allergens via the oral mucosa during sublingual immunotherapy. Int Arch Allergy Immunol 1999; 120: 218-24.

26. Fenoglio D, Puppo F, Cirillo I, et al. Sublingual specific immunotherapy reduces PBMC proliferations. Allergol Immunol 2005; 37: 147-51.

27. Ciprandi G, Fenoglio D, Cirillo I, et al. Sublingual HDM-specific immunotherapy induces IL-10 production: preliminary report. Ann Allergy Asthma Immunol 2005; 95; 38-44.

28. Ciprandi G, Fenoglio D, Cirillo I, Milanese M, Minut P. Sublingual Immunotherapy and Tregs. Allergy 2006; 61: $511-3$.

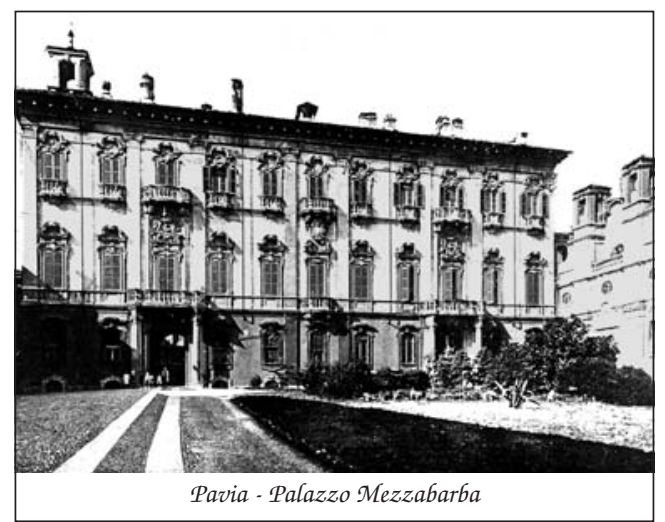

